ESAIM: PROCEEDINGS, April 2012, Vol. 36, p. 180-188

Danièle Fournier-Prunaret, Laura Gardini, \& Ludwig Reich, Editors

\title{
DOUBLING BIFURCATION OF A CLOSED INVARIANT CURVE IN 3D MAPS
}

\author{
LAURA Gardini ${ }^{1}$ AND IRYNA SUShKO ${ }^{2}$
}

\begin{abstract}
The object of the present paper is to give a qualitative description of the bifurcation mechanisms associated with a closed invariant curve in three-dimensional maps, leading to its doubling, not related to a standard doubling of tori. We propose an explanation on how a closed invariant attracting curve, born via Neimark-Sacker bifurcation, can be transformed into a repelling one giving birth to a new attracting closed invariant curve which has doubled loops.
\end{abstract}

AMS (2000) subject classification. 37G15, 37G35, 37C70.

Keywords: 3D maps, Neimark-Sacker bifurcation, closed invariant curve, period-doubling bifurcation.

Résumé. L'objet de ce papier est de donner une description qualitative des mécanismes de bifurcation associés avec une courbe fermée invariante pour des applications en dimension trois, conduisant à son doublement, et différent du doublement standard de tores. Nous proposons une explication du fait qu'une courbe fermée invariante attractive, née d'une bifurcation de Neimark-Sacker, peut se transformer en une courve répulsive donnant naissance à une nouvelle courbe fermée invariante attractive avec boucles doublées.

Mots clefs: Transformation de dimension 3, Bifurcation de Neimark-Sacker, Courbe invariante fermée, Bifurcation doublement de période.

\section{INTRODUCTION}

In several applied models (see, e.g., $[1,3-5])$ it has been observed that closed invariant curves in three dimensional (3D for short) maps can undergo a kind of doubling bifurcation sequence. The bifurcation mechanism leading to such a dynamic behavior was announced as an open problem at the European Conference on Iteration Theory in 2006 (ECIT-06) [6]. The observed phenomenon consists in the following: a closed invariant attracting curve, born via Neimark-Sacker (NS for short) bifurcation from a fixed point of a 3D map, is transformed into a repelling curve surrounded by a new attracting closed invariant curve which is doubled in shape. A cascade of such bifurcations can be observed, that is, the new attracting curve, in its turn, can undergo the same kind of bifurcation.

As an example, in Fig.1 we show the closed invariant attracting curve and a sequence of the bifurcations mentioned above in the Lotka-Volterra model represented by the 3D map

$$
\begin{aligned}
& x^{\prime}=x+R x(1-x-a y-b z) \\
& y^{\prime}=y+R y(1-b x-y-a z) \\
& z^{\prime}=z+R z(1-a x-b y-z)
\end{aligned}
$$

\footnotetext{
${ }^{1}$ Dept. of Economics, Society and Politics, University of Urbino, Italy; e-mail: laura.gardini@uniurb.it.

${ }^{2}$ Institute of Mathematics, NASU; Kyiv Economics Institute at Kyiv School of Economics; e-mail: sushko@imath.kiev.ua.
}

(c) EDP Sciences, SMAI 2012 
where the parameters $R$ and $a$ are fixed as $R=1, a=0.5$, and $b$ is varied (taken from [5] and [3]). Our aim is to give a qualitative description of the possible mechanism of such bifurcations. Although no rigorous proof is given, our arguments are consistent with the dynamics and explain the observed phenomena. The properties we are going to describe are based on the geometrical structure of the invariant sets associated with closed invariant curves. In our opinion, this doubling phenomenon is associated with a period doubling bifurcation of a pair of cycles belonging to the invariant closed curves. The case of ergodic (or quasiperiodic) dynamics has been recently considered in [2].
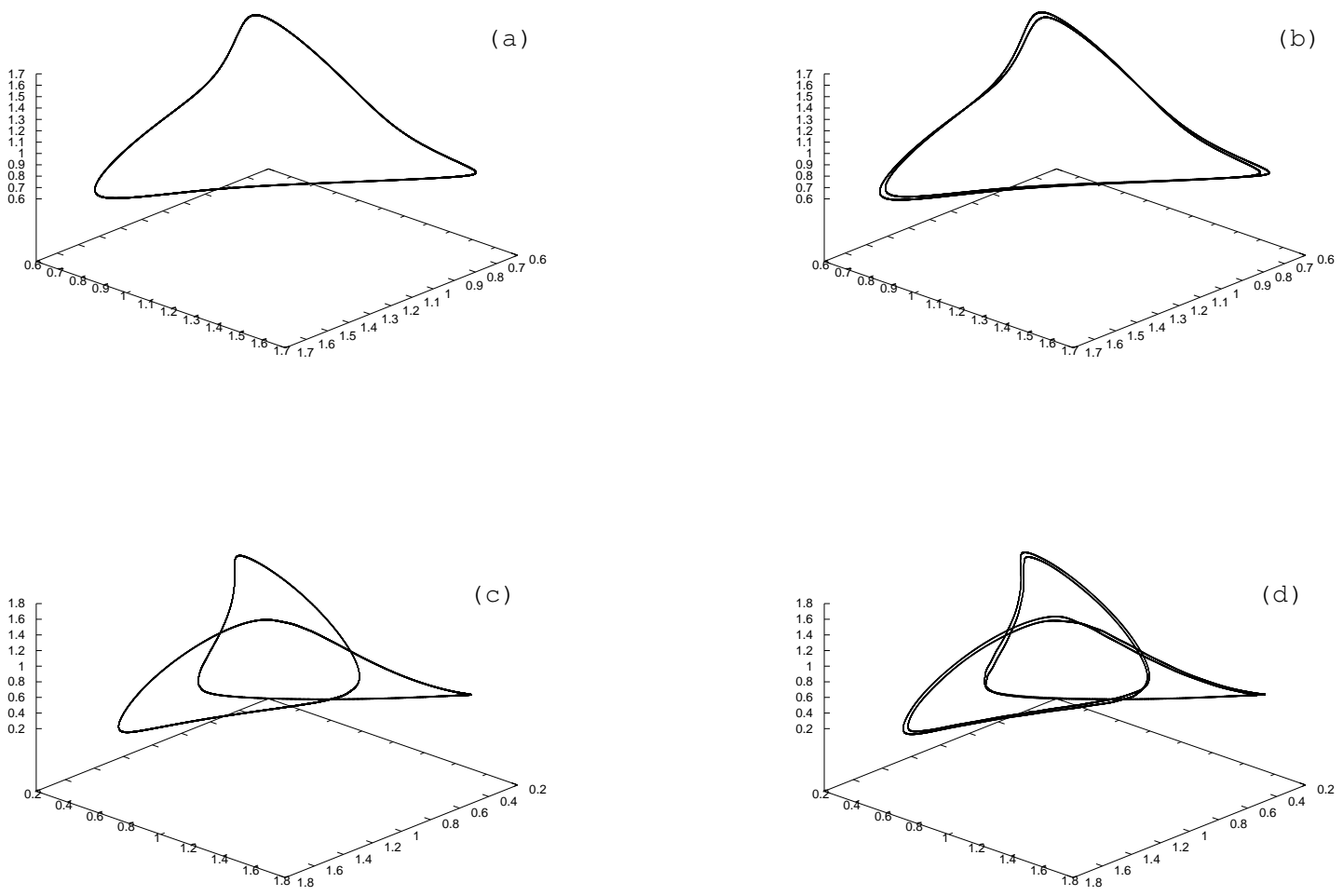

Figure 1. Attractors of the 3D Lotka-Volterra map with $R=1$ and $a=0.5$ fixed. In (a) $b=-0.657$, closed curve born via Neimark-Sacker bifurcation of the fixed point $P^{*}=(\xi, \xi, \xi)$, $\xi=1 /(1+a+b)$; in (b) and (c) first period doubling in the range $b \in[-0.65735,-0.65736]$; in (d) second period doubling in the range $b \in[-0.666,-0.667]$. A third period doubling occurs in the range $b \in[-0.6687,-0.6688]$

The plan of the work is as follows. In the next section we recall in short the mechanism of the NS bifurcation in $2 \mathrm{D}$ maps. It necessarily involves the two complex conjugate eigenvalues of the Jacobian evaluated at the bifurcating fixed point. The 3D case is considered in Section 3. Obviously, the third eigenvalue of the fixed point undergoing the NS bifurcation, is necessarily real and in general it is positive or negative. As we shall see, the sign of the third eigenvalue plays an important role in the bifurcations which involve the attracting closed invariant curve $\Gamma$ appearing after the NS bifurcation and surrounding the unstable fixed point.

In short, the bifurcation which we study consists in the following. Consider two cycles, one attracting node and a saddle, whose saddle-node connection constitutes the closed invariant attracting curve. Let the third 
eigenvalue of these cycles, related to the eigenvectors transverse to the $2 \mathrm{D}$ manifold including the fixed point and the invariant curve, pass through -1 (in general, it occurs not simultaneously for both cycles but consequently). This leads to the creation of a 2D invariant manifold which may be a normal strip or a Möbius strip: The manifold includes the repelling closed curve $\Gamma$ and two disjoint closed invariant attracting curves if the eigenvalue transverse to the manifold is positive, while if this eigenvalue is negative then a unique closed invariant attracting curve exists, which constitutes the boundary of a Möbius strip. So, in section 3 we describe our conjecture in the case of an attracting closed invariant curve associated with a supercritical NS bifurcation, distinguishing between two cases, that is, Case I leading to two disjoint closed invariant curves and Case II leading to a unique closed invariant curve on the boundary of a Möbius strip.

\section{The Neimark-Sacker Bifurcation in a 2D maP}

In this section we briefly recall the mechanism of the NS bifurcation which a fixed point (focus) $P^{*}$ of a $2 \mathrm{D}$ map undergoes, and describe some properties of a closed invariant attracting curve surrounding the unstable focus after the NS bifurcation. Although such a bifurcation is very common, the dynamics of the map restricted to the closed invariant curve are not so well known, so, we recall also a few related facts.

Consider a 2D map. Let it depend on two parameters $a$ and $b$. It is well-known that in the $(\tau, \delta)$-plane, where $\tau$ is trace and $\delta$ is determinant of the Jacobi matrix of the 2D map, the conditions of the local stability of the fixed point form a so-called stability triangle with sides $\delta=1, \delta=\tau+1$ and $\delta=-\tau-1$. Then the NS bifurcation occurs when the parameters $a$ and $b$ vary in such a way that the side $\delta=1$ of the stability triangle is crossed. In the $(a, b)$-parameter plane the condition $\delta=1$ in general corresponds to a curve. We are interested in the occurrence of a supercritical NS bifurcation. It is well known that from the NS bifurcation curve infinitely many periodicity regions are issuing, the so-called Arnold tongues, related to different rotation numbers of the corresponding cycles of the map, as qualitatively shown in Fig.2a. Each periodicity region is associated with the existence of at list two cycles, a saddle and attracting node, whose saddle-node connection constitutes an attracting closed invariant curve, say $\Gamma$, born due to the NS bifurcation, as schematically shown in Fig.2b. The closed invariant curve $\Gamma$ is transversely attracting, and the dynamics of the map, restricted to the $1 \mathrm{D}$ invariant set $\Gamma$ are such that a generic trajectory is attracted to the stable cycle (except for the points of the saddle cycle).

If the 2D map represents the first return map in a $2 \mathrm{D}$ phase plane of a Poincaré section $\Sigma$ of a $3 \mathrm{D}$ flow, then the stable fixed point $P^{*}$ corresponds to the intersection of a stable limit cycle $G$ of the flow with the plane $\Sigma$ (in Fig.2c only a portion of the limit cycle $G$ is shown). After the NS bifurcation of $P^{*}$ the attracting closed invariant curve $\Gamma$ is the intersection with $\Sigma$ of an attracting torus $T$ existing in the 3D phase space (see Fig.2d), which surrounds the unstable limit cycle $G$. When the closed curve $\Gamma$ is given by a saddle-node connection then the generic trajectory on the torus $T$ is attracted to a limit cycle belonging to the torus, which intersects the Poincaré section at the periodic points of the attracting cycle on $\Gamma$. On the torus $T$ an unstable limit cycle also exists, which intersects the Poincaré section at the periodic points of the saddle cycle on $\Gamma$. For this reason in the theory of continuous time dynamical systems the described dynamic behavior is commonly known as 'phase locked' or 'frequency locked' case.

When a parameter path in the parameter space is, for example, as indicated by the green arc in Fig.2a, then infinitely many Arnold tongues are crossed. The sequence of bifurcations leading from one periodicity region to a different one are well known, at list for parameter values not far from the NS bifurcation curve. Namely, the boundaries of the periodicity regions, issuing from the NS bifurcation curve, correspond to the saddle-node bifurcations leading to merging and disappearance (or appearance) of the pair of cycles on $\Gamma$. The points from which the periodicity regions are issuing correspond to rational rotation numbers, which are maintained inside the periodicity regions (for a definition of rotation numbers and other details see, e. g., [8]).

The other kind of dynamics on the closed invariant curve $\Gamma$ is a quasiperiodic behavior: any trajectory forms a dense set on the closed curve $\Gamma$. Quasiperiodic dynamics on $\Gamma$ correspond to irrational rotation numbers, and if we consider the corresponding flow then the related trajectories are dense on the torus $T$. 


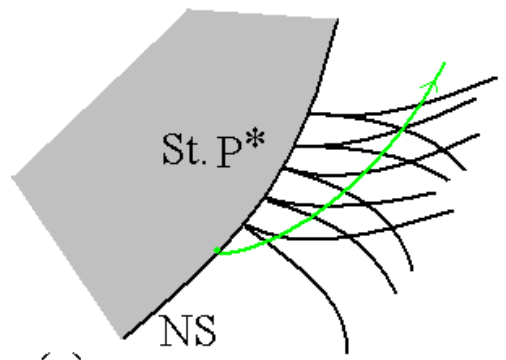

(a)

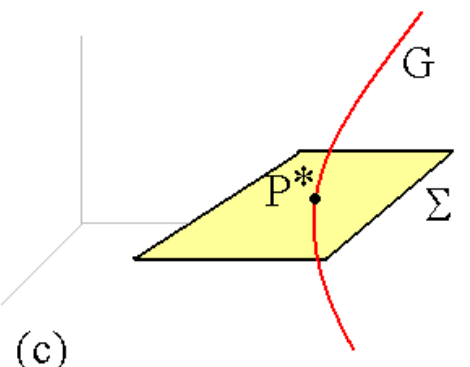

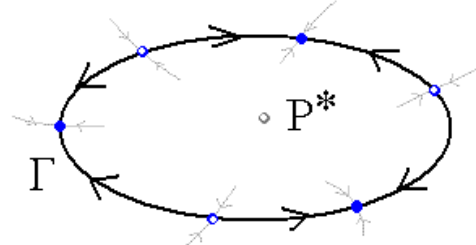

(b)

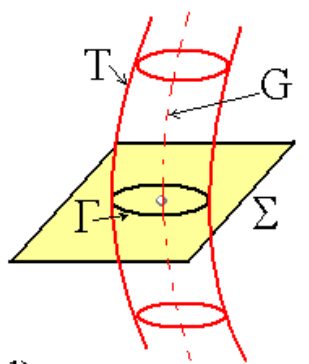

(d)

Figure 2. Qualitative pictures.

In the parameter plane the periodicity regions are organized according to the so-called adding rule, also referred to as Farrey summation rule, applied to the related rotation numbers. In short, it consists in the following: between any two periodicity regions related to the rotation numbers, say, $m_{1} / n_{1}$ and $m_{2} / n_{2}$, there exists a periodicity region related to the rotation number $\left(m_{1}+m_{2}\right) /\left(n_{1}+n_{2}\right)$. Note that the rational rotation is not generic only for parameter values taken exactly on the NS bifurcation curve, while soon after the bifurcation the rational rotation becomes generic: infinitely many periodicity regions fill the parameter plane densely. The parameter sets corresponding to quasiperiodic dynamics also exist after the NS bifurcation but these sets are only curves, not regions (see also the description of the periodicity regions for a piecewise smooth map in [9]). This is the reason why for a parameter path crossing the periodicity regions as, e. g., the green arc in Fig.2a, the related diagram 'rotation number versus parameter' has a form of devil staircase: the periodic trajectories correspond to nonempty segments, which are dense on a generic parameter path issuing from NS bifurcation curve. This fact is associated with structural stability, that is, the map is structurally stable when the parameters correspond to a rational rotation number, while it is structurally unstable when the dynamics are quasiperiodic (see $[8])$.

Thus, the generic behavior of a trajectory on a closed invariant curve $\Gamma$ is a saddle-node case, and the related cycles are the invariant sets responsible for the property of stability of the invariant set $\Gamma$. In fact, the bifurcations of the curve $\Gamma$ are studied via the bifurcations occurring to the cycles on it as, for example, the homoclinic bifurcation leading to destruction of the closed invariant curve.

The properties mentioned above related to the NS bifurcation in 2D maps, and, in particular, to the bifurcation structure of the parameter space, persist in the case of a NS bifurcation in 3D maps, which is closely related to the main object of our study.

\section{The NeIMARK-SACKER BIFURCATION in A 3D MAP}

Let us turn now to a 3D map $M$, assuming that its fixed point (focus) $P^{*}$ undergoes a NS bifurcation. We can reason, to fix the ideas, using a 2D parameter plane so that the NS bifurcation curve is as qualitatively shown in Fig.2a. The Jacobian matrix evaluated at the fixed point $P^{*}$ has a pair of complex conjugate eigenvalues 

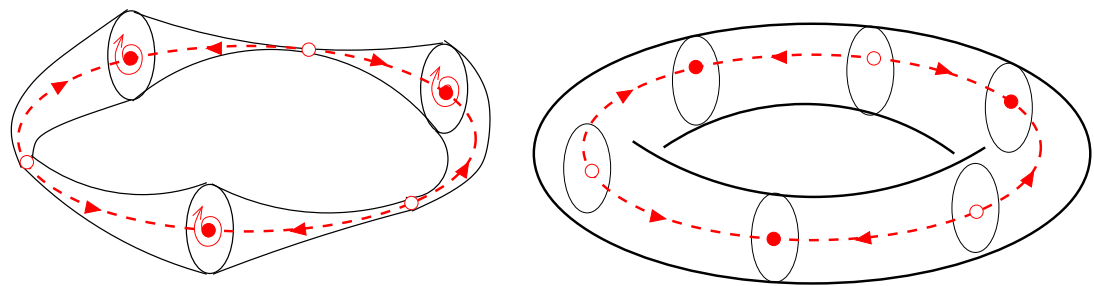

FiguRE 3. Qualitative picture associated with the bifurcation of the closed invariant curve via complex eigenvalues.

$\lambda_{1}$ and $\lambda_{2}$, whose modulus becomes larger then 1, while a third real eigenvalue exists, say $\lambda_{3}$, and let $\left|\lambda_{3}\right|<1$. When this third real eigenvalue has a sign then it is responsible for two different bifurcation scenarios. We shall consider this later. For the moment we note that after the NS bifurcation of $P^{*}$, which we assume here of supercritical type, an attracting closed invariant curve $\Gamma$ appears, surrounding the unstable fixed point $P^{*}$ (see, for example, Fig.1a). And also, even if the phase space is 3D instead of 2D, the closed invariant set is given in general by the saddle-node connection of a pair of cycles, an attracting node denoted $C_{N}$ and a saddle denoted $C_{S}$. In fact, the pair of eigenvalues $\lambda_{1,2}$ are inherited by the pair of cycles on $\Gamma$ similarly to what occurs in a 2D map (we recall that any point on the NS bifurcation curve associated with a rational rotation is also the merging of two saddle-node bifurcation curves), while the third real eigenvalue of the cycles on $\Gamma$ can be assumed approximately close to $\lambda_{3}$.

\subsection{Complex eigenvalues}

Then, due to the existence of three eigenvalues, it is also possible to have a pair of complex eigenvalues of the cycles on the closed invariant curve, and several related bifurcations have been studied. For example, in the case of a complex pair the NS bifurcation of the cycle $C_{N}$ gives rise to the appearance of a closed invariant torus-like surface, but with shrinking points in the saddle $C_{S}$ (see the qualitative picture in Fig. $3 \mathrm{a}^{1}$ ). If also the cycle $C_{S}$ undergoes a NS bifurcation then a 3D torus appears, which is attracting, and it includes the old closed invariant curve $\Gamma$, now unstable, in its interior (see the qualitative picture in Fig.3b). We do not know if such a 3D torus may be associated with a torus in a flow at least 4D. Several other cases and instructive examples can be found in [7].

\subsection{Real eigenvalues}

However, the bifurcations which we are interested in are associated with real eigenvalues. So, let us assume that the cycles belonging to $\Gamma$ have all real eigenvalues, say $\lambda_{j}^{N}$ and $\lambda_{j}^{S}$ (with $j=1,2,3$ ) for the node and saddle, respectively, where $j=3$ gives the one associated with the eigenvalue $\lambda_{3}$ of $P^{*}$.

Obviously, the doubling of the closed invariant curve $\Gamma$ must be associated with flip bifurcations of cycles on $\Gamma$ (the node $C_{N}$ and the saddle $C_{S}$ ). We know that one of the three eigenvalues of both cycles must be positive (say $\lambda_{1}^{N}>0$ and $\lambda_{1}^{S}>0$ ), and the related manifolds give the branches of the saddle-node connection and, thus, constitute the closed invariant curve. Let us assume now that the second eigenvalue of both cycles becomes negative (say $\lambda_{2}^{N}<0$ and $\lambda_{2}^{S}<0$ ), and that both approach the bifurcation value -1 . We remark that it is not necessary that the flip bifurcations of $C_{N}$ and $C_{S}$ occur simultaneously. As both have values close to each other it is more realistic to argue that, as some parameter is varied, one bifurcation occurs first and then the second one. We notice that in an applied model it may be quite difficult to observe the doubling of a closed curve when the related cycles have a low period. On the other hand, when it is associated with cycles of a high period, the period doubling bifurcations of the two cycles occur almost simultaneously, so that the effect can be numerically observed, but it is difficult to detect the related period.

\footnotetext{
${ }^{1}$ We thank Soumitro Banerjee for this figure.
} 
Clearly the node becomes a saddle, and an attracting node of double period $C_{2 N}$ appears, with periodic points on both sides of the unstable manifold issuing from $C_{N}$. Similarly for the saddle, a second branch becomes unstable, and a saddle of double period $C_{2 S}$ appears, with periodic points on both sides of the unstable manifold issuing from $C_{S}$. We know that close to the bifurcation value, the new periodic points of double period appear very close to those which are bifurcating, and after the second flip bifurcation also manifolds connecting the saddle $C_{2 S}$ with the attracting node $C_{2 N}$ must exist associated with the positive eigenvalue of both cycles, inherited from the positive eigenvalues $\lambda_{1}^{N}>0$ and $\lambda_{1}^{S}>0$, and the related branches of manifold issuing from the new saddle $C_{2 S}$ will reach the new attracting node $C_{2 N}$. Thus, the second flip bifurcation leads the invariant curve $\Gamma$ to become repelling (but still existing as invariant curve) while a new attracting closed invariant curve must be created, say $\Gamma_{2}$, and an invariant manifold exists which includes both the curves, the unstable $\Gamma$ and the stable $\Gamma_{2}$.

What is the shape of the new invariant manifold created via this pair of flip bifurcations? The answer is not unique. Indeed we have still not taken into account the third eigenvalue of the cycles, $\lambda_{3}^{N}$ and $\lambda_{3}^{S}$ (which both have in general the sign inherited from the eigenvalue $\lambda_{3}$ of the fixed point $\left.P^{*}\right)$. Above we required $\left|\lambda_{3}\right|<1$ without any specific requirement on the sign. Let us consider the sign of the third eigenvalue $\lambda_{3}^{N}$ and $\lambda_{3}^{S}$. Then the two cases positive or negative give two qualitative different bifurcations associated with the doubling of the closed invariant curve $\Gamma$, that is, with the appearance of $\Gamma_{2}$. In fact, if the third eigenvalue $\left(\lambda_{3}^{N}\right.$ and $\left.\lambda_{3}^{S}\right)$ is positive (case I), then we have trajectories converging to the stable node approaching only one side of the related invariant manifold, and we have an invariant set as qualitatively drawn in Fig.4a. While if the third eigenvalue $\left(\lambda_{3}^{N}\right.$ and $\left.\lambda_{3}^{S}\right)$ is negative (case II), then we have trajectories converging to the stable node approaching both sides of the related invariant manifold, and we have an invariant set as qualitatively drawn in Fig.4b. Let us describe in more details the two cases.
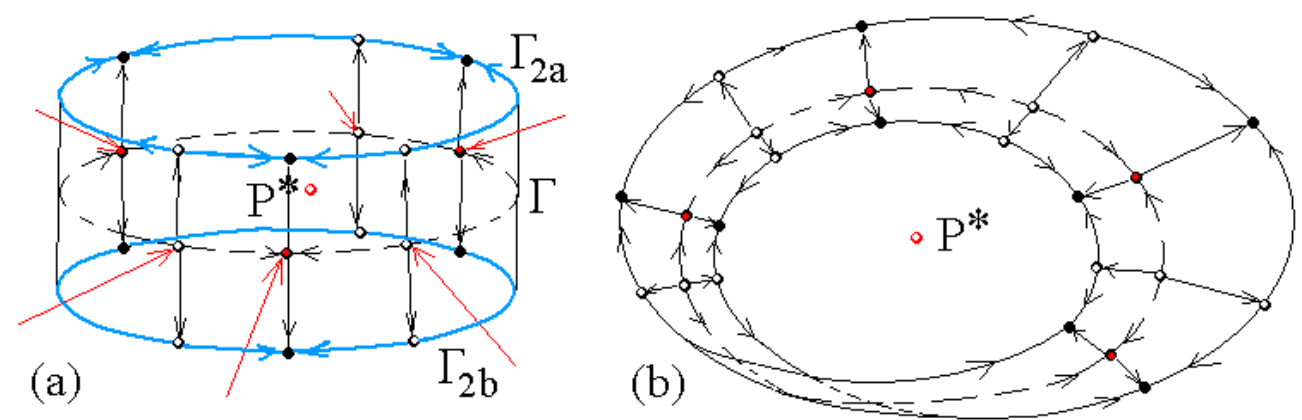

FiguRE 4. Qualitative picture associated with the bifurcation of the closed invariant curve via flip bifurcations, with third eigenvalue positive in (a) and negative in (b).

Case I $\left(\lambda_{3}^{N}>0\right.$ and $\left.\lambda_{3}^{S}>0\right)$. If the third eigenvalue is positive then we have that the new attracting set $\Gamma_{2}$ consists of two disjoint invariant curves $\Gamma_{2}=\Gamma_{2 a} \cup \Gamma_{2 b}$, which bound a strip (or manifold) having qualitatively the shape of a portion of cylinder. This manifold includes the unstable curve $\Gamma$ and the invariant manifolds connecting the bifurcated cycles $C_{N}$ and $C_{S}$ with those of double period born at the flip bifurcation of $C_{2 N}$ and of the saddle $C_{2 S}$. Due to the dynamic behavior associated with the flip bifurcation, on the invariant curve $\Gamma_{2}$ the points jump from $\Gamma_{2 a}$ to $\Gamma_{2 b}$ and vice versa, from $\Gamma_{2 b}$ to $\Gamma_{2 a}$. The strip is a simple orientable manifold, and the two components $\Gamma_{2 a}$ and $\Gamma_{2 b}$ are disjoint sets, although cyclically invariant, as in fact they are single closed invariant curves for the second iterate $M^{2}$ of the map.

This bifurcation can be associated with the torus doubling bifurcation in a flow at list 4D. That is, this 3D map may be the Poincaré section of a flow. Then the first closed curve $\Gamma$ represents the section of a torus $T$ of the flow and the bifurcation of the closed curve $\Gamma$ giving rise to the two disjoint closed curves, cyclically visited, 
represents the bifurcation of torus doubling. The two disjoint components $\Gamma_{2 a}$ and $\Gamma_{2 b}$ are the two sections of a doubled torus, as roughly illustrated in Fig. $5^{2}$.
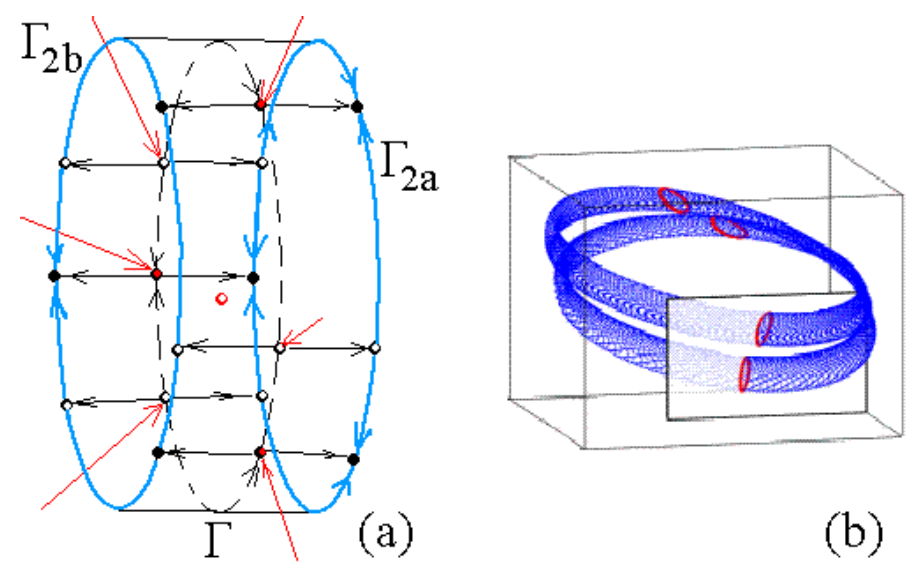

(b)

Figure 5. Qualitative picture of a Poincaré section in the case of third eigenvalue positive in (a), and the related doubled torus in (b).

Qualitatively the same bifurcation in the 3D phase space of the map, that is two disjoint closed curves, say $\Gamma_{a}$ and $\Gamma_{b}$, cyclically invariant for the map, may also be created following a different bifurcation path. In fact, starting from the stable fixed point, if the destabilization of $P^{*}$ occurs via a flip bifurcation of a real eigenvalue, then it becomes a saddle and a stable two cycle is created (see the qualitative pictures in Fig.6). This 2-cycle may undergo a NS bifurcation through the other pair of eigenvalues, assumed complex.

This sequence of local bifurcations, first flip of $P^{*}$ and then NS of the 2-cycle, also leads to a pair of cyclically invariant closed curves $\Gamma_{a}$ and $\Gamma_{b}$ (Fig.6c), but the invariant set which includes the two curves is not a regular strip, and due to the lack of a repelling closed invariant curve $\Gamma$ in between, this bifurcation cannot be associated with the period doubling of a torus in a flow.

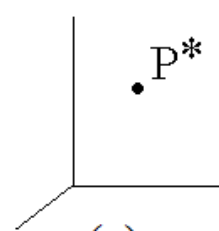

(a)

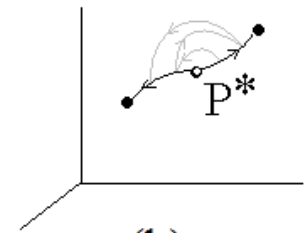

(b)

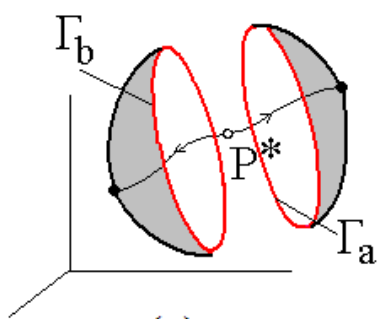

(c)

FiguRE 6. Qualitative picture of a stable fixed point in (a), its flip bifurcation in (b), and the supercritical NS bifurcation of the 2-cycle in (c). No closed curve exists.

Case II $\left(\lambda_{3}^{N}<0\right.$ and $\left.\lambda_{3}^{S}<0\right)$. In the other case, characterized by the third eigenvalue of negative sign (so that all the cycles, $C_{N}, C_{S}, C_{2 N}$ and $C_{2 S}$ have a pair of negative eigenvalues), we must have that the trajectories are approaching the stable node jumping in some way from the interior of the strip to the exterior of it, and the invariant set is a non-orientable strip, so that it has now the shape of a Möbius strip, the border of which is the closed invariant curve of double period, which we have observed in many examples, one of which

\footnotetext{
${ }^{2}$ Fig.5b was taken from the thesis of H.G.E. Meijer (2009) downloadable from the web site.
} 
is reported in the Introduction, and shown in Fig.1b (see also Fig.1c). The qualitative geometric configuration is also shown in Fig. $7^{3}$.

The closed curve $\Gamma_{2}$ qualitatively shown in Fig.4b and Fig.7 is the boundary of the Möbius strip. Notice that now $\Gamma_{2}$ is invariant also for the second iterate of the map. We do not know if now the border of the Möbius strip may be associated with some kind of torus in a flow at list $4 \mathrm{D}$, for example something like a twisted torus.

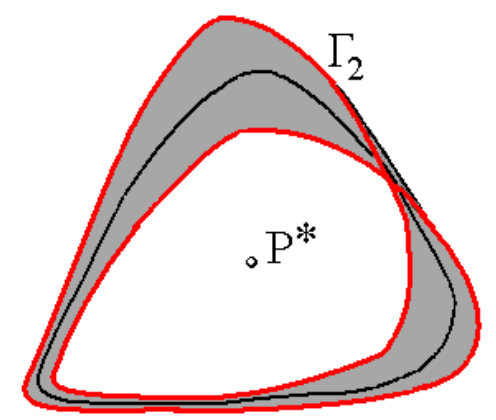

FIgURE 7. Qualitative figure of the closed invariant curve $\Gamma_{2}$.

We remark again that such invariant sets, the orientable strip in case I and the Möbius strip in case II, are attracting for the $3 \mathrm{D}$ map $M$, and on them the attractor consists in the two disjoint closed curves $\Gamma_{2 a} \cup \Gamma_{2 b}$ in case I, and the unique closed doubled curve $\Gamma_{2}$ in case II.

As already remarked in the Introduction, the occurrence of a period doubling of the closed invariant curve may be repeated, and now the mechanism is clearly the same, applied to the cycles giving the saddle-node connection of the closed invariant curve. It is known that the flip bifurcations may occur in cascade, and this indeed was observed in our example in Fig.1. Fig.1d shows a closed curve $\Gamma_{4}$ doubled from $\Gamma_{2}$, and as the parameter $b$ is further decreased we have also observed a further doubling, a closed curve $\Gamma_{8}$ doubled from $\Gamma_{4}$ (not shown in the figure because the attracting curve is very close to the unstable one and the layers are not well distinguished unless with a strong enlargement).

The closed invariant curve is then broken. In our example this probably occurs when an attracting node on the closed curve undergoes a flip bifurcation which destroys the closed curve, leading quickly to chaos via a sequence of period doubling bifurcations.

It is important to emphasize that for the example given shown in Fig.1, in the large interval of values of the parameter $b$ for which a closed invariant curve exists (simple or doubled, from $\Gamma$ to $\Gamma_{8}$ ) one can observe the crossing of several periodicity regions. That is, the cycles leading to period doubling of the curves change as a function of the parameter. They may merge and disappear, quasiperiodic motion can exist, and then again the periodic one: the dynamic behavior is exactly of the same kind that we observe also in 2D maps.

\section{REFERENCES}

[1] V. Avrutin, P. Levi, M. Schanz, D. Fundinger and G. Osipenko, Investigation of Dynamical Systems Using Symbolic Images: Efficient Implementation and Applications, Int. J. Bifurcation and Chaos, 16(12), 2006, 3451-3496.

[2] S. Banerjee, D. Giaouris, Local Bifurcations of a Quasiperiodic Orbit, Int. J. Bifurcation and Chaos, 2011 (to appear).

[3] G.I. Bischi, F. Tramontana, Three-dimensional discrete-time Lotka-Volterra models with an application to industrial clusters, Communications in Nonlinear Science and Numerical Simulations 15, 2010, 3000-3014.

[4] D. Fournier-Prunaret, R. Lopez.Ruitz, A.K. Taha, Route to chaos in three-dimensional maps of logistic type, Proceedings of the Conference ECIT-04, Grazer Mathematische Berichte N.350, 2006, 82-95.

\footnotetext{
${ }^{3}$ Fig.7 was inspired by a figure from "Dynamical Systems, Graphs, and Algorithms", G. Osipenko, Springer Verlag, N.Y., 2007.
} 
[5] L. Gardini, R. Lupini, C. Mammana, M.G. Messia, Bifurcations and transitions to chaos in the three-dimensional LotkaVolterra map, SIAM J. Appl. Math., 47, 1987, 455-482.

[6] L. Gardini, Period-Doubling bifurcation of closed invariant curves in three-dimensional maps, in G.L. Forti, D. Gronau, L. Paganoni, L. Reich, J. Smital(Eds.), Grazer Math. Ber., ISSN 1016-7692 Bericht Nr. 351, 2007, Section Problems and Remarks, 181-182.

[7] G. Osipenko, Dynamical Systems, Graphs, and Algorithms, Springer Verlag, Berlin, 2007.

[8] Y.A. Kuznetsov, Elements of applied bifurcation theory, Springer-Verlag, New-York, 1998.

[9] D.J.W. Simpson, J.D. Meiss, Neimark-Sacker Bifurcations in Planar, Piecewise-Smooth, Continuous Maps, SIAM J. Applied Dynamical Systems, 7(3), 2008, 795-824.

[10] I. Sushko, L. Gardini, Center Bifurcation for Two-Dimensional Border-Collision Normal Form, Int. J. Bifurcation and Chaos, 18(4), 2008, 1029-1050. 